\section{Bevölkerungsweite Studie zu Tamoxifen bei DCIS}

Seit einer durchschlagenden Studie aus dem Jahr 1999 bekamen Frauen mit duktalem Karzinom in situ (DCIS) zusätzlich zu brusterhaltender Operation und Bestrahlung auch Tamoxifen. Später zeigte sich, dass vor allem die Frauen mit positivem Östrogenrezeptor(ER)-Status profitieren. Kanadische Forscher untersuchten nun, ob die Erkenntnisse allgemeingültig sind.

B eim DCIS hatte Tamoxifen 1999 in einer Studie des National Surgical Adjuvant Breast and Bowel Projects (NSABP-B24) das Rezidivrisiko bei DCIS-Patientinnen gesenkt, wenn diese sowohl brusterhaltend operiert als auch bestrahlt worden waren [Fisher B et al. Lancet. 1999;353(9169):1993-2000]. 2002 zeigte eine Subgruppenanalyse (die Allred-Studie), dass vor allem Frauen mit Östrogenrezeptor(ER)-positivem Tumor von einer endokrinen Therapie (ET) profitieren [Allred DC et al. J Clin Oncol. 2012;30(12):1268-73].

Nun wurde untersucht, inwieweit sich das auf dem Bevölkerungslevel bestätigen lässt. Zwischen 1989 und 2009 er- hielten 2.061 Frauen mit DCIS eine brusterhaltende Therapie plus Bestrahlung, 417 in der Prä-NSABP-B24-Ära (1989 bis 1998) und 1.548 in der Post-NSABP-B24Ära (2000 bis 2009). Die zweite Kohorte wurde weiter unterteilt in die Prä- und Post-Allred-Ära. Eine ET wurde bei 404 Patientinnen (20\%) eingesetzt. Dass mediane Alter der ET-behandelten Frauen lag bei 53 gegenüber 57 Jahren in der Gesamtkohorte $(\mathrm{p}<0,0005)$. In der PräNSABP-B24-Ära bekam 1/417 Patientinnen $(0,2 \%)$ eine ET, in der Post-NSABPB24-Ära waren es 399/1548 (26\%). Nach der Allred-Studie wurden 227 Tumoren mit einer ET behandelt; von diesen waren $65 \%$ ER-positiv, $1 \%$ ER-negativ und bei $33 \%$ war der ER-Status unbekannt. 801 Patientinnen erhielten keine ET; von diesen waren $43 \%$ ER-positiv und $15 \%$ ER-negativ, $42 \%$ hatten einen unbekannten ER-Status ( $\mathrm{p}<0,0005)$.

Eine multivariate Analyse der PostNSABP-B24-Ära zeigte, dass eine ET mit einem längeren krankheitsfreien Überleben (DFS) der DCIS-Patientinnen verbunden war (Hazard Ratio 0,6; $\mathrm{p}=$ $0,02)$; die Rate betrug mit ET nach 5 Jahren 96,9 versus $94,5 \%$ ohne ET.

Fazit: Der Einsatz von ET bei DCIS-Patientinnen, die brusterhaltende Operation und Bestrahlung bekamen, steigerte sich nach der NSABP-B24-Studie, vor allem bei positivem ER-Status und jüngerem Alter. Die ET verbesserte zudem das DFS. Die Daten von NSABP-B24 und Allred lassen sich somit bevölkerungsweit generalisieren.

Christina Berndt

Lo AC et al. Population-based analysis of the impact and generalizability of the NSABP-B24 study on endocrine therapy for patients with ductal carcinoma in situ of the breast. Ann Oncol. 2015;26(9):1898-903.

\title{
Brustkrebs: Breitere Ränder bei partieller Mastektomie sind besser
}

\begin{abstract}
Die großzügigere Resektion der Ränder der Operationshöhle könnte die Häufigkeit von positiven Rändern und Reexzisionen bei Brustkrebspatientinnen, die sich einer partiellen Mastektomie unterziehen, reduzieren.
\end{abstract}

\footnotetext{
- twa 20-40\% der Patientinnen, bei de- nen eine partielle Mastektomie durchgeführt wurde, müssen sich einer Nachresektion der Schnittränder unterziehen. Retrospektive Studiendaten zeigten, dass die großzügigere Entnahme von Gewebe in der Umgebung der Kavität die Rate positiver Ränder reduzieren könnte. Andererseits wird argumentiert, dass die Standardresektion ausgewählter Ränder, an die der Tumor nah heranreicht, ausreichend ist. In einer prospektiv randomisierten Studie wurde die breitere Resektion der Ränder verglichen mit der Resektion ausgewähl-
}

ter Ränder. 235 Patientinnen mit Brustkrebs (Stadium 0-III) wurden nach Ende der partiellen Standardmastektomie intraoperativ randomisiert, eine weitere Resektion der Kavitätenränder (Shave-Gruppe) oder keine weitere Resektion (No-Shave-Gruppe) zu erhalten.

Das mediane Alter der Patientinnen lag bei 61 Jahren. Bei der letzten pathologischen Untersuchung hatten $23 \%$ von ihnen einen invasiven Tumor, $19 \%$ ein duktales Karzinoma in situ (DCIS), $53 \%$ beides. Die mediane Größe des Tumors im größten Durchmesser betrug bei $\mathrm{Pa}$ tientinnen mit invasivem Tumor $1,1 \mathrm{~cm}$ $(0-6,5 \mathrm{~cm})$, bei denen mit DCIS $1,0 \mathrm{~cm}$ $(0-9,3 \mathrm{~cm})$.

Die Häufigkeit positiver Ränder nach der partiellen Mastektomie und vor der Randomisierung war in beiden Gruppen vergleichbar (36 bzw. 34\%; p =0,69). Nach der Randomisierung hatten die $\mathrm{Pa}$ tientinnen in der Shave-Gruppe signifikant seltener positive Ränder (primärer
Endpunkt) als diejenigen in der NoShave-Gruppe (19 vs. $34 \%$; $=0,01$ ). Außerdem war in der Shave-Gruppe signifikant seltener eine zweite Operation zur Entfernung positiver Ränder erforderlich (10 vs. $21 \%$; p = 0,02). Das kosmetische Ergebnis beurteilten die Patientinnen in den beiden Gruppen vergleichbar. Bezüglich der Komplikationen gab es keinen signifikanten Unterschied zwischen den beiden Gruppen.

Fazit: Die großzügigere Resektion der Kavitätenränder halbierte die Rate positiver Ränder und erneuter Exzisionen bei Patientinnen, die sich einer partiellen Mastektomie unterzogen - ohne, dass Abstriche bei den kosmetischen Ergebnissen gemacht werden mussten.

Judith Neumaier

Chagpar AB et al. A Randomized, Controlled Trial of Cavity Shave Margins in Breast Cancer. N Engl J Med. 2015;373(6):503-10. 